\title{
ESTRÉS Y SU AFRONTAMIENTO EN DIRECTORES Y DIRECTORAS DE COLEGIOS Y ESCUELAS EN EL CIRCUITO 03 DE LA PROVINCIA DE HEREDIA
}

\author{
Pedro Ureña Bonilla' \\ Carmen Castro Sancho
}

El propósito de este estudio fue analizar la problemática de la vivencia del estrés y su afrontamiento en directores y directoras del circuito 03 de la provincia de Heredia. Se contó con la participación de 17 administradores educativos, entre ellos, 11 mujeres y 6 hombres. Los instrumentos utilizados permitieron valorar situaciones estresantes, correlato emocional propio de cada situación, perfiles anímicos y estrategias de afrontamiento. En general los resultados encontrados permiten la identificación de factores importantes como disparadores de la vivencia del estrés y del acompañante emocional. Entre ellos, la irresponsabilidad, la falta de compromiso y de identificación con la causa institucional, la pérdida de control y el irrespeto entre otros. Asimismo, el perfil animico mostró sobre todo, a un grupo de profesionales con una buena reserva de vitalidad, a pesar de la carga que representa el puesto. Finalmente, las estrategias de afrontamiento utilizadas son predominantemente de corte racional y hacen referencia también a un grupo de directores y directoras que, ante la vivencia de estrés, prefieren planificar sus acciones, actuar en función del problema y reflexionar sobre la situación.
The purpose of this study was to analyze how school principals working in the third circuit of the province of Heredia experienced and faced stress. 17 educational managers ( 11 women and 6 men) participated in the survey. The instruments used allowed us to assess stressing situations, the emotional correlation of each one of the situations, mood profiles and strategies to face them. In general, the findings allowed important factors to be identified as triggers of stress and its accompanying emotions. Some of these factors are: irresponsibility, lack of commitment and of a sense of belonging to the institution, lack of control and lack of respect. The psychological profile basically revealed a group of professionals with good vitality in spite of the burden derived from their positions. Finally, the strategies to cope with stress are mainly rational and denote a group of principals that prefer to plan their actions, to respond according to the problem and to reflect upon the situation.

1 Doctor en Ciencias del Deporte con especialidad en Psicología Deportiva, por la Universidad de Deportes, Colonia, Alemania. Licenciado en Educación Física por la Escuela Ciencias del Deporte de la Universidad Nacional. Actualmente decano de la Facultad de Ciencias de la Salud.

2 Máster en Administración Educativa. Directora del Colegio Técnico Profesional de Flores. También ha impartido cursos a nivel universitario en áreas propias de la administración y planificación educativa. 
Asociado con la viencia del

estrés se reporta con

frecuencia, la presencia de

cefaleas, cáncer, depresión, entre otras situaciones graves, aunque también se reporta otra serie de alteraciones que afectan la calidad de vida de las personas, entre ellas, la desconcentración, el descontrol emocional, el insomio, los problemas de apetito, las malas relaciones sociales, asociado todo ello con ausencias periódicas e incapacidades frecuentes, en fin con una mala calidad de vida

\section{Introducción}

La vivencia de estrés y el proceso de afrontamiento del mismo están imbricados en el contexto transaccio-nal persona - medio (Lazarus \& Folkman, 1986). En este marco, la percepción y valoración subjetiva de las personas es fundamental. De tal forma, el que una situación particular genere la vivencia de estrés dependerá de la forma en que la persona en cuestión interprete dicha situación (Lazanus y Folkman 1986). A I respecto es importante decir que, la persona es parte de la situación y la juzga desde una perspectiva inclusiva (Nitch, 1986). Así, una situación cualquiera puede percibirse e interpretarse como un reto, como una amenaza o como un daño (Lazarus y Folkman, 1986). Por supuesto que, en todo este proceso valorativo, la instancia persona es fundamental en la medida en que, es a partir de su propia subjetividad que se desarrolla el proceso del estres. Dan contenido al marco valorativo e interpretativo de la personal, sus experiencias, su presente y sus expectativas (Nitch, 1986). Asimismo, la vivencia y afrontamiento del estrés se puede manifestar en mayor o menor medida en la parte somática, en la psicológica, particularmente en la dimensión emocional - afectiva y cognitiva y finalmente en la dimensión conductual (Weimberg y Could, 2000). De manera particular Hackfort y Schwenkmesger (1955) destacan algunos elementos que son fundamentales como disparadores de distintas emociones; así por ejemplo mencionan que las situaciones - temor, se caracterizan por representar una amenaza, incertidumbre, incontrolabilidad e impredecibilidad y porque en ellas se vislumbra el fracaso.

Por otro lado, para Kuhl y Schulz (1996) las situaciones - enojo, se dan cuando de ellas se infiera un obstáculo que impide el logro de una meta que se creía alcanzada; o cuando en un contex to transaccional se perciba que se afectan intereses y necesidades personales, o cuando haya que modificar planes de acción debido a obstáculos inesperados (Isberg, 2000). También el nerviosismo como forma previa de temor o miedo se presenta cuando no se sabe si la meta propuesta será alcanzada o no (Jul y Schulz,1986). Valga subrayar que, en el 
modelo que proponemos, las emociones tienen una función reguladora en los procesos transaccionales individuo medio (Hackfort, 1987, Nitch, 1986). De acuerdo con lo expuesto hasta aqui, entenderíamos el estrés como un proceso que tiene lugar en el psicofísico (Nitch, 1986) con consecuencias de menor o mayor envergadura para los implicados en el contexto de transacción particular.

El afrontamiento fonma parte de fenómeno de la vivencia del estrés y representa todo el conjunto de esfuerzos cognitivos y conductuales (Lazarus y Folkman, 1986) que se implementan en situaciones de desequilibrio psicofísico. En este proceso, la personal puede asumir una posición pasiva, activa o de evitación (método), también puede focalizarse en atender el problema o bien la emoción y finalmente, proceder cognitivamente o de manera conductual (Femández, 2000). Por supuesto que, la intensidad de la vivencia del estrés y la frecuencia con que se experimente, son fundamentales como elementos explicativos de las consecuencias que se asocian con este fenómeno. Asimismo, juegan un papel básico los mecanismos de afrontamiento que la persona utilice (Sonnenschein, 1995). Asociado con la viencia del estrés se reporta con frecuencia, la presencia de cefaleas, cáncer, depresión, entre otras situaciones graves, aunque también se reporta otra serie de alteraciones que afectan la calidad de vidade las personas, entre ellas, la desconcentración, el descontrol emocional, el insomio, los problemas de apetito, las malas relaciones sociales, asociado todo ello con ausencias periódicas e incapacidades frecuentes, en fin con una mala calidad de vida (Femández, 1998, Simón, 1997). De manera que, en cualquier contex to transaccional individuo - medio podria generarse la vivencia de estrés y en consecuencia activarse procesos de afrontamiento como parte del mismo fenómeno (Lazanus y' Folkman, 1986). El espacio transaccional que se activa en el desempeño de la labor administrativo-académica se caracteriza por una serie de elementos que favorecen la vivencia del estrés y que atentan contra el desempeño eficiente de la ocupación y finalmente contra una buena calidad de vida. Dado el tipo de proyecto que está en manos de un director o directora de escuela o colegio, a decir, la fonmación integral de los ciudadanos y ciudadanas del mañana, no es de extrañar la existencia de una serie de elementos inherentes al oficio del administrador educativo, que sin lugar a dudas, es caldo de cultivo para la vivencia del estrés. Es claro que, el abordaje de esta problemática representa un aporte fundamental en la medida en que permitirá un diagnóstico aproximado de un problema existente en el diario vivir de los directores y directoras de colegios y escuelas $y$, cuyas consecuencias son de gran trascendencia y por consiguiente hay que enfrentar. En este sentido, la promoción de una buena calidad de vida en los profesionales que ejercen el oficio de la dirección académico-administrativa en los niveles de primaria y secundaria, debcría constituirse en una meta fundamental, primero por la salud de los mismos y segundo porque le permitirán asumir su función de líder con más eficiencia y eficacia. 
En el marco de lo expuesto en los párrafos anteriores, cl propósito fundamental de este trabajo es analizar la problemática de la vivencia de estrés en el contexto transaccional típico de los administradores educativos. De manera específica se trata de identificar situaciones en que usualmente el director o directora de colegio o escuela experimenta la vivencia de cstrés. Asimismo, se pretende determinar el conelato emocional propio de la vivencia de estrés en cada una de las situaciones caracterizadas como estresoras. Finalmente, se propone tipificar perfiles anímicos e identificar mecanismos de afrontamiento.

\section{Metodología}

El estudio se realizó con un grupo de 17 directores y directoras de escuclas y colegios, pertenecientes al circuito 03 de la provincia de Heredia. El grupo estuvo conformado por 6 hombres y 11 mujeres, con edad promedio de 41 años. El tiem. po promedio de ejercer como administradores en instituciones educativas es de 9.3 años. Los profesionales supracitados contestaron 3 instrumentos diseñados para recopilar información sobre situaciones estresantes, correlato emocional propio de la vivencia del cstrés, estados de ánimo y mecanismos de afrontamiento.

El primer instrumento consta de 15 ítemes que hacen referencia a situaciones propias del espacio transaccional construido en el ejercicio de la función de administrador educativo. Estas situaciones fucron seleccionadas a partir de conversaciones informales con un grupo de directores y directoras de colegio y escuela, quienes coincidieron en lo representativo de las mismas como situaciones estresantes. El valor estresante de cada situación es determinado a partir de una escala de 5 opciones, donde: $1=$ no me genera estrés, $2=$ me genera poco estrés, $3=$ medianamente me genera estrés, 4= me genera bastante estrés y $5=$ me genera mucho estrés. En el segundo instrumento se valora el correlato emocional ante la vivencia de cada una de las mismas 15 situaciones juzgadas en la primera escala. En este caso se cuenta con las siguientes categorías de respuesta: l= Enojado, furioso, molesto, histérico, 2= Triste, impotente, desilusionado, frustrado, $3=$ Agotado, exhausto, liquidado y $4=$ Incómodo, ansioso, tenso, nervioso. Para la tipificación del estado de ánimo se utilizó una versión modificada del Perfil de Estados Anímicos (Profile of Mood State) (Bal aguer, Meliá y García, 1996). A través de estacscala podemos evaluar sentimientos, afectos y estados de ánimo (LeUnes y Hayıvard, 1989; Ávila y Jiménez, 1991. Este instrumento consta de 29 ítemes y está compuesto por los factores: depresión, el que representa un estado anímico deprimido acompañado de sentimientos de inadecuación personal y está definido por adjetivos como: desdichado, infeliz, desamparado, amargado, triste y deprimido. El factor hostilidad se refiere a un estado anímico de cólera y antipatía hacia los demás. Los adjetivos que lo componen son: enfado, enojado, de mal genio, furioso, 
molesto e initable. El factor tensión está caracterizado, por adjetivos que describen aumentos en la tensión músculo esquelético: inquieto, intranquilo, nervioso, tenso, relajado y agitado. El factor vigor hace rcferencia a un estado de vigor, ebullición y energía elevada. Lo describen los siguientes adjetivos: enérgico, lleno de energía, vigoroso, activo, luchador y animado. El factor fatiga representa un estado anímico de laxitud, inercia y bajo nivel de energía. Formado por los adjetivos: fatigado, agotado, cansado, exhausto y sin fuerzas.

\section{Cuadro 1}

Factores e ítems correspondientes a la escala Perfil de estados anímicos

\begin{tabular}{ll}
\hline Depresión & Items $3+6+13+16+23+26$ \\
Hostilidad & Items $4+9+14+19+24+29$ \\
Tension & Items $1+8+11+18+21+(28$ puntúa Negativo $)$ \\
Vigor & Items $2+7+12+17+22+27$ \\
Fatiga & Items $5+10+15+20+25$ \\
\hline
\end{tabular}

El tercer instrumento utilizado fue el inventario de estilos y estrategias de afrontamiento (Femández, 2000). El propósito de este cuestionario es recopilar información sobre el conjunto de esfuerzos cognitivos y conductuales que se implementan durante la vivencia del estrés (Lazarus y Folkman, 1986). Consta de 54 ítems y permite el reconocimiento de estrategias y estilos de afrontamiento. Utiliza una escala de 4 opciones: nunca $=0$, a veces $=I$, bastante $=2$ y siempre $=3$. Dado que son tres ítems los que corresponden a cada estrategia, el máximo puntaje es de 9, con un valor promedio de 4.5. Las estrategias de afrontamiento se obtienen de la suma de las puntuaciones dadas a los ítems propios de cada estrategia.

\section{Cuadro 2}

\section{Estrategias e ítems respectivos del Inventario de estilos y estrategias de afrontamiento}

\section{Estrategias}

\section{items}
A) Reevaluación positiva
B) Reacción depresiva
C) Negación
D) Planificación
E) Conformismo
F) Desconexión cognitiva
G) Desarrollo personal
H) Control emocional

$15+25+33$

$32+44+63$

$1+4+38$

$18+29+51$

$5+37+47$

$17+20+50$

$3+19+49$

$39+43+48$ 
I) Distanciamiento

$6+34+41$

J) Supresión de actividades distractoras

$9+13+45$

K) Refrendar el afrontamiento

$10+21+31$

L) Evitar el afrontamiento

$8+11+26$

M) Resolver el problema

$22+35+42$

N) Apoyo social al problema

$7+14+24$

N) Desconexión comportamental

$27+30+36$

O) Expresión emocional $40+46+53$

P) Apoyo social emocional $12+16+23$

Q) Respuesta paliativa

$2+38+54$

Entre mayor sea la puntuación obtenida en cada estrategia, significa que más habitualmente se utiliza esa estrategia de afrontamiento.

La identificación de estilos se realiza a partir de la apreciación de las estrategias, sumando el valor correspondiente a cada una de ellas según se indica en el cuadro siguiente.

\section{Cuadro 3}

Clasificación por método, focalización y actividad del Inventario de estilos y estrategias de afrontamiento

\section{Método}

Activo

$\mathrm{A}+\mathrm{D}+\mathrm{G}+\mathrm{J}+\mathrm{M}+\mathrm{O}$

Pasivo

$\mathrm{B}+\mathrm{E}+\mathrm{H}+\mathrm{K}+\mathrm{N}+\mathrm{P}$

Evitación

$\mathrm{C}+\mathrm{F}+\mathrm{I}+\mathrm{L}+\tilde{\mathrm{N}}+\mathrm{Q}$

\section{Focalización}

Respuesta

Problema

$\mathrm{A}+\mathrm{B}+\mathrm{C}+\mathrm{J}+\mathrm{K}+\mathrm{L}$

Emoción

$\mathrm{D}+\mathrm{E}+\mathrm{F}+\mathrm{M}+\mathrm{N}+\tilde{\mathrm{N}}$

$\mathrm{G}+\mathrm{H}+\mathrm{I}+\mathrm{l}+\mathrm{P}+\mathrm{Q}$

Actividad

Cognitiva

$\mathrm{A}+\mathrm{B}+\mathrm{C}+\mathrm{D}+\mathrm{E}+\mathrm{F}+\mathrm{G}+\mathrm{H}+\mathrm{I}$

Conductual

$$
\mathrm{J}+\mathrm{K}+\mathrm{L}+\mathrm{M}+\mathrm{N}+\tilde{\mathrm{N}}+\mathrm{O}+\mathrm{P}+\mathrm{Q}
$$


Paracadauno de los estilos de afrontamiento del bloque de método y focalización, se obtendrá una puntuación que oscilará entre $O$ y 54 , con un valor promedio de 27 , mientras que para el bloque de actividades obtendrá una puntuación que oscilará entre 0 y 81 con un valor promedio de 40.5. Cuanto mayor sea la puntuación obtenida significa que más habitualmente se utiliza ese estilo de afrontamiento.

Los instrumentos fueron aplicados en forma grupal y en presencia de los investigadores lo que permitió aclarar dudas a la hora de contestar los mismos.

Finalmente, los análisis estadísticos utilizados implicaron el uso de técnicas descriptivas, correlativas e inferenciales. Todos los cálculos se realizaron utilizando el paquete estadístico para las ciencias sociales (SPSS).

\section{Resultados}

Con el propósito de determinar el valor estresante de cada situación identificada como propia del contexto transaccional de directoras y directores se calcularon valores promedio. El análisis arrojó los siguientes resultados.

\section{Cuadro 4}

Apreciación del valor estresante de situaciones escogidas

\section{Situaciones}

1. Los profesores no entregan la información que se les pide a tiempo

2. Me solicitan informes de un día para otro

3. El inicio de los ciclos lectivos

4. El cierre de los ciclos lectivos

5. Atender a los padres de familia

6. Conducir reuniones con profesores

7. Los estudiantes se quejan de la pedagogía del profesor

8. Los profesores no empiezan las clases puntualmente

9. Los profesores no tienen control de la clase

10. El personal evade responsabilidades argumentando que eso no les toca

11. Los profesores dejan salir a los alumnos antes de la hora

12. Las ausencias periódicas de profesores y administrativos sin previo aviso

13. Las malas relaciones entre el personal Pronedio

14. Cambio de políticas educativas constantes

15. Los exámenes de bachillerato o de sexto
3.35

1.70

2.92

3-56

$3.66(4)$

2.64

1.92 
Tal y como se puede observar en el cuadro anterior, los directores y directoras participantes en este estudio, valoran como situaciones con mayor contenido estresante: (1) EL PERSONALEVADE RESPONSABILIDADES ARGUMENTANDO QUE ESO NO LES TOCA; (2) ME SOLICITAN INFORMES DE UN DIA PARA OTRO; (3) LOS PROFESORES NO TIENEN CONTROL DE LA CLASE; (4) AUSENCIAS PERIÓDICAS DEL PERSONAL SIN PREVIO AVISO Y (5) LOS PROFESORES NO ENTREGAN LA INFORMACIÓN QUE SE LES PIDE A TIEMPO. Como poco estresantes son apreciadas las situaciones: EL INICIO DE LOS CICLOS LECTIVOS; CONDUCIR REUNIONES CON LOS PROFESORES $Y$ LOS EXAMENES DE BACHILLERATO O SEXTO.

Por otro lado el análisis relativo al correlato emocional propio de la vivencia de estrés en cada una de las situaciones valoradas como estresantes mostró los siguientes resultados:

\section{Cuadro 5}

\section{Correlato emocional ante situaciones estresantes}

\section{Situaciones}

Cat-A Cat-B Cat-C Cat-D

1. Los profesores no entregan la

información que se les pide a tiempo

2. Me solicitan informes de un día para otro

3. El inicio de los ciclos lectivos

4. El cierre de los ciclos lectivos

5. Atender a los padres de familia

6. Conducir reuniones con profesores

7. Los estudiantes se quejan de la pedagogía del profesor

8. Los profesores no empiezan las clases puntualmente

9. Los profesores no tienen control de la clase

10. El personal evade responsabilidades argumentando que eso no les toca

11. Los profesores dejan salir a los alumnos antes de la hora

12. Las ausencias periódicas de profesores y administrativos sin previo aviso

13. Las malas relaciones entre el personal

14. Cambio de políticas educativas constantes

15. Los exámenes de bachillerato o de sexto

$\begin{array}{llll}3 & 4 & 2 & 8 \\ 2 & 6 & 1 & 6 \\ 1 & 0 & 7 & 7 \\ 0 & 1 & 4 & 6 \\ 0 & 2 & 1 & 5 \\ 0 & 2 & 1 & 5 \\ 1 & 4 & 0 & 4 \\ 4 & 5 & 0 & 5 \\ 5 & 3 & 1 & 4\end{array}$

$\begin{array}{llll}7 & 3 & 0 & 6\end{array}$

$\begin{array}{llll}4 & 3 & 0 & 6\end{array}$

$\begin{array}{llll}6 & 2 & 2 & 4\end{array}$

$\begin{array}{llll}1 & 5 & 1 & 8\end{array}$

$4 \quad 2 \quad 1 \quad 6$

$\begin{array}{llll}0 & 0 & 1 & 7\end{array}$

Cat-A=Enojado, furioso, molesto, histérico.

Cat-B=Triste, impotente, desilusionado, frustrado.

Cat-C=Agotado, exhausto, liquidado.

Cat. $\cdot \mathrm{D}=$ Incómodo, ansioso, tenso, nervioso. 
De la lectura del cuadro anterior resalta como hecho predominante, que el acompañante emocional con mayor frecuencia mencionado corresponde a la categoría Cat-D (INCÓMODO, ANSIOSO, TENSO Y NERVIOSISMO). La excepción se observa en las situaciones: LOS PROFESORES NO TIENENCONTROL DE LA CLASE, EL PERSONAL EVADE RESPONSABILIDADES ARGUMENTANTO QUE ESO NO LES TOCA Y LAS AUSENCIAS PERIÓDICAS DE PROFESORES Y ADMINISTRATIVOS SIN PREVIO AVISO, en que el correlato emocional preponderante es representado por la categoría ENOJADO, FURIOSO, MOLESTO, HISTÉRICO. Asimismo, llama la atención las situaciones: ME SOLICITAN INFORMES DE UN DIA PARA OTRO Y LOS PROFESORES NO EMPIEZANLAS CLASES PUNTUALMENTE, en que el acompañante emocional TRISTE, IMPOTENTE, DESILUSIONADO, FRUSTRADO, ocupa el mismo rango que la Cat-D. Asimismo, la situación EL INICIO DE LOS CICLOS LECTIVOS, ocupan igual rango en cuanto a correlato emocional, la categoría AGOTADO, EXHAUSTO, LIQUIDADO y la Cat-D.

Asimismo, el análisis general del perfil anímico -emocional característico de directores y directoras, mostró los siguientes valores.

\section{Cuadro 6}

Perfil anímico correspondiente a la semana anterior a la realización del presente estudio

\begin{tabular}{l|c}
\hline Factores anímicos & Puntajes promedio \\
\hline Depresión & 3.75 \\
Hostilidad & 6.29 \\
Vigor & 12.92 \\
Fatiga & 7.36 \\
Tensión & 9.55 \\
\hline
\end{tabular}

La información contenida en el cuadro anterior nos permite identificar sobre todo, niveles realmente bajos en relación con el factor DEPRESIÓN. En orden ascendente aunque con poca importancia se encuentm los factores HOSTILIDAD, FATIGA y TENSIÓN. Destaca sobre todo, el valor superior correspondiente al factor VIGOR, el que sc caracteriza por ítems como: enérgico, lleno de energía, vigoroso.

En cuanto al análisis de las estrategias de afrontamiento utilizadas por directores y directoras se encontraron los siguientes resultados. 


\begin{tabular}{l|r}
\hline Estrategias de afrontamiento & Puntajes promedio \\
\hline A. Reevaluación positiva & $7.27(4)$ \\
B. Reacción depresiva & 3.60 \\
C. Negación & 3.87 \\
D. Planificación & $7.62(3)$ \\
E. Conformismo & 6.25 \\
F. Desconexión cognitiva & 4.72 \\
G. Desarrollo personal & $8.30(1)$ \\
H. Control emocional & 5.56 \\
I. Distanciamiento & 5.82 \\
J. Supresión de actividades distractoras & 5.66 \\
K. Refrendar el afrontamiento & 6.37 \\
L. Evitar el afrontamiento & 5.11 \\
M. Resolver el problema & $7.66(2)$ \\
N. Apoyo social problema & 5.46 \\
O. Expresión emocional & 5.25 \\
P. Apoyo social emocional & 6.07 \\
Q. Respuesta paliativa & 4.45 \\
\hline
\end{tabular}

La lectura del cuadro antcrior nos permite identificar las cstrategias mayormente utilizadas para hacer frente al estrés, cn orden descendentc:

EIDESARROLLO PERSONAL, dimensión que incluye ítems como-picnso que la experiencia aporta cosas positivas (3), intento aprender de todas las cxpcricncias (19) y me sirve para descubrir lo que es realmente importante cn la vida (49). RESOLVER EL PROBLEMA, conformado por los ítems: me responsabilizo en hacer algo para rcsolver el problema (22), tomo todas las medidas para que las cosas marchen bien (35) y aúno todos los esfucrzos para resolver el problema (45). PLANIFICACIÓN, dimensión integrada por los ítems: reflexiono sobre cuál es la mejor manera de resolvcr el problema (18), recurro a experiencias anteriores para encontrar una solución (29) y preparo un plan para actuar (56) y finalmente la REEVALUACIÓN POSITIVA, conformada por los ítemes: mc propongo cambiar la forma en que veía el problema (15), me empeño en sacar los aspectos positivos (25) y procuro darle la vuelta a los problemas para 
ver lo más positivo (32), me cuesta mucho hacer frente a los problemas por mí mismo (47) y espero a que cambien las cosas (63). La NEGACIÓN, conformada por los ítems: me niego a creer en lo que ha ocurrido (1), desfiguro el problema (4) y fantaseo para evitar pensar en lo que ha sucedido (28) y RESPUESTA PALIATIVA, conformada por los ítems: busco alivio a mi situación en la religión (2), intento acallar mis emociones comiendo, fumando o tomando medicamentos (40) y evito mis sentimientos mediante la bebida o el consumo de drogas (66).

Resumiendo el proceso de afrontamiento según método, focalización y actividad nos encontramos con el siguiente resultado.

\section{Cuadro 8}

Método, focalización y actividad en el proceso de afrontamiento

\begin{tabular}{lc} 
Método & Valor promedio \\
\hline Activo & 37.05 \\
Pasivo & 28.67 \\
Evitación & 25.68 \\
Focalización & \\
Respuesta & \\
Problema & 27.45 \\
Emoción & 34.85 \\
& 31.67 \\
Actividad & \\
Cognitiva & \\
Conductual & $\mathbf{4 9 . 1 3}$ \\
\hline
\end{tabular}

El análisis de la información contenida en el cuadro anterior nos hace evidente que el método de afrontamiento predilecto es el activo, asimismo que, los esfuerzos de focalización se orientan preponderantemente hacia el problema y que la actividad que se asume en el enfrentamiento del estrés, es tanto de orden cognitivo como conductual.

El análisis comparativo por sexo, para el caso de la valoración de situaciones estresantes, no mostró diferencias significativas $(p>.05)$. Tanto los directores como las directoras coinciden en cuanto a la valoración que hacen de las 
situaciones juzgadas. Tampoco se diferencian de manera significativa ( $p>.05)$ en cuanto al acompañante emocional vivido en cada situación cstresantc. En . clación con el perfil anímico, los hombres en comparación con las mujeres mostraron un índice de fatiga significativamente supcrior $(t=2.54 ; \mathrm{p}=.04)$. Por otra parte a propósito de las estrategias de afrontamiento, se encontraron diferencias significativas entre hombres y mujeres en los factores: PLANIFICACIÓN $(1=2.34 ; p=.04)$ y REFRENDAR EL AFRONTAMIENTO ( $t=2.55 ; p=.02)$. En ambos casos, los hombres en comparación con las mujeres tienden a utilizar con más frecuencia csas estrategias para afrontar el estrés. A pesar de estas diferencias en torno de las estrategias, en cuanto al método, la focalización y la actividad, no se registraron diferencias significativas entre hombres y mujercs ( $p>.05$ ).

Asimismo, el análisis corrclativo entrc los diversos factores estudiados mostró para el caso de las dimensiones del perfil emocional, correlaciones positivas y significativas entre HOSTILIDAD Y DEPRESIÓN $(r=.59 ; p>.05)$, HOSTILIDAD Y TENSIÓN $(r=.52 ; p<.05)$, TENSIÓN Y FATIGA $(r=.77: p$ $<.05)$, DEPRESIÓN Y TENSIÓN ( $r=.66$; $p<.05)$.

También; se encontraron correlaciones positivas y significativas entre las dimensiones: MÉTODO ACTIVO Y FOCALIZACIÓN EN EL PROBLEMA ( $r$ $=.95) ; p<.05)$, MÉTODO ACTIVO Y ACTIVIDAD CONDUCTUAL ( $r=.78$; $p<, 05)$, MÉTODO DE EVITACIÓN Y ACTIVIDAD CONDUCTUAL $(r=$ $.67 ; p<.05)$, MÉTODO DE EVITACIÓN Y MÉTODO PASIVO $(r=.75 ; p<$ .05), MÉTODO DE EVITACIÓN Y ACTIVIDAD COGNITIVA $(r=.80 ; p<$ .05), FOCALIZACIÓN EN EL PROBLEMA Y ACTIVIDAD CONDUCTUAL $(r=.74: p<.05)$, ACTIVIDAD CONDUCTUAL Y MÉTODO PASIVO $(r=$ $.62 ; p<.05)$ y MÉTODO PASIVO Y ACTIVIDAD COGNITIVA $(r=.79 ; p<$ .05). Finalmente, se encontró una correlación significativa y positiva entre el factor FATIGA correspondiente al perfil anímico y la dimensión MÉTODO ACTIVO propio de las estratcgias de afrontamiento $(r=.66 ; p<.05)$.

\section{Discusión}

El propósito de este estudio fue analizar la problemática de la vivencia del estrés y su afrontamiento en el caso de directores y directoras de instituciones educativas de primaria y secundaria. Los resultados nos permiten identificar en primera instancia, que las situaciones que disparan con mayor intensidad la vivencia del estićs, se caracterizan por la fácil lectura de elementos como la irresponsabilidad, la falta de compromiso e identificación con un proyecto que se supone común, el irrespeto a un ordenamiento establecido, la rendición de cuentas, ambiente de grupo negativo y la falıa de planificación o improvisación. 
Estos son aspectos que, en criterio de Lazarus y Folkman (1986), representan una amenaża para el cumplimiento de las metas que toda institución de enseñanza, bajo la conducción no es de extrañar que generen la vivencia de estrés. Sin embargo, no hubo diferencias significativas entre hombres y mujeres en cuanto a la valoración hecha sobre el valor estresante de las situaciones juzgadas.

De manera particularizada, nos encontramos con una predominancia de la categoría emocional: "enojado, furioso, molesto, histérico" (Cat-A), en situacioncs que dejan inferir contextos transaccionales en que sc podría fácilmentc interpretar; falta de compromiso, poca identificación con la causa, poca solidaridad, irresponsabilidad, irrespeto, falta dc orden e incapacidad. Valoraciones que se asocian con la vivencia de estados emocionales como los reportados Isberg (2000), Kuhl y Schulz (1996) y Hackfort (1987).

Asimismo, la preponderancia de la catcgoría emocional: "incómodo, ansioso, tenso, nervioso" (Cat-D), se obscrvó en situaciones que hacen obvio aspectos como: la negligencia, irresponsabilidad, falta de compromiso, anticipación de mucho trabajo, anticipación de discusiones poco constructivas, falta de control, desorden, malas interrclaciones, incertidumbrc y mala planificación. Elementos que, de acuerdo con Ranglin y Hanin (2000), Kuhl y Schulz (1996) y Hackfort (1987), se asocian con los correlatos emocionales mencionados.

Las categorías emocionales: "Incómodo, ansioso, tenso, nervioso" (CatD) y "triste, impotente, desilusionado, frustado" (Cat-B), compartieron rango y se caracterizaron por ser evocadas en situaciones en que los dircctorcs y dircctoras se ven obligados a cambiar su foco de atención para atender demandas urgentes de instancias superiores, antc las cualcs hay poco tiempo de acción. Además en situaciones en donde sc vislumbra el fracaso, sc siente falta de compromiso, incumplimiento del deber, la pérdida de control de los profesorcs por un lado y la nccesidad de control de los directores y dircctoras. Estos resultados también coinciden con las apreciaciones de Ranglin y Harin (2000), Kuhl y Schulz (1996) y llackfort (1987).

Para todos los casos anteriores en que sc exploró el correlato emocional, no hubo di ferencias significativas entre directores y directoras. Este hecho nos permite inferir un marco intcipretativo similar, a la hora de hacer la lectura de los contextos transaccionales implicados en cada una de las situaciones valoradas (Lazarus y Folkman, 19S6) y Nitsh, 1986). Por ejemplo, es de esperar en ambos casos, tanto expectativas como mctas similares, lo quc daría contexto a un marco de interprctación común.

El pcrfil anímico mostró, sobre todo, a un grupo de directores y directoras entusiastas y cargados de vitalidad, a pesar de la carga que cl pucsto representa. Esto podria estar asociado, al mismo tipo de afirontamicnto que tipifica el proccso 
de la vivencia del estrés en estas personas. Sin embargo, las mujeres en comparación con los hombres mostraron mayores índices de tolerancia a la fatiga, evidenciado en items como: lleno de energía, luchador, animado. Coincidente con Kuhl y Schulz (1996) a pesar de no encontrarse indices altos en los factores correspondientes al perfil anímico, si encontramos que entre mayor sea la hostilidad mayor tension, a mayor tension mayor fatiga y a mayor tension, mayores índices de depresión. Estas relaciones dejan ver con gran claridad la importancia de atender el perfil anímico como un correlato más de la vivencia de estrés.

Asimismo, entre las estrategias de afrontamiento con mayor frecuencia utilizadas por el grupo de directores y directoras participantes en este estudio, destacan aquellas que tienen que ver con la planificación, la focalización en el problema, la reflexión y la acción. Estamos, de acuerdo con Fernández (2000), ante un perfil de afrontamiento positivo y saludable. Sin duda, se trata de un grupo de profesionales que, dadas las características de su rol, ante todo racionaliza y actúa sin rehuir la situación que genera estrés.

En terminos generales, tanto los directores como las directoras, afrontan de manera similar el estres, sin embargo, los hombres en comparación con las mujeres muestran una mayor tendencia a reflexionar y planificar el afrontamiento, a darse tiempo para resolver; situación que se hace evidente en las respuestas cmitidas ante ítems como: reflcxiono sobre cuál es la mejor manera de resolver el problema, preparo un plan para actuar, me contengo hasta saber más sobre el problema. También, el método de evitación como alternativa de afrontamiento se asocia con la nccesidad de actuar haciendo otras cosas ajenas a la situación estresante y con la actividad cognitiva no vinculada. Finalmentc y como era de esperarse, la necesidad de tomar acciones en el proceso de afrontamiento se vincul6 de manera significativa con los indices de fatiga que experimentan directores y directoras.

\section{Referencias}

Balagucr, I. Mclía, J.L. y García-Meritá (1993)- El perfil de los cstados de ánimo POMS). Revisı de Psicología del Deporle, No. 4, pp. 39-52.

Fernández - Abascal, E. (2002). Estilos y cstratcgias de afrontamiento. En E. Fernández - Abascal. F. Palmcro; M.Ch6liz y F. Martínez. (Eds.). Cuaderno de prácticas y de motivación y emoción. Barcelona: Pirámide, pp. 189-206. 
Fernández - Abascal, E. (1998), Emociones y a alaptación. Barcelona:Ariel.

Hackfort, D. y Emotion. In H. Eberspacher (HG) Handlexikon der Sportwissenkschafi. Rowohit: Rororo, pp. 88-98.

Hackfort, D. y Schwenkmezger, P. (1980). Angst und angstkontrolle im Sport. Koln: bps.

Isberg, L. (2000). Anger, Agressive Behavior, and Athletic Perfomance. En Y. Hanin (Ed.) Emotions in Sport. Champaign: Human Kinetics, pp. 113-135.

Kuhl, U. y Schulz, P. (1996). Emotionale Belastung im Sport. Koln:bps.

Lazarus, R. y Folkman, S. (1986) Estrés y procesos cognitivos. Barcelona: Martínez Roca.

Nitsch, J. (1986). Zur handlungstheoretischen Grundlegung der Sportpsychologic. In H. Gabler; J.R. Nitsh \& R. Singer (Hrgs.) Einfuhrung in die Sportpsychologie. Schomdorf: Hofmann, pp. 188-267.

Ranglin, J. Hanin, Y. (2000), Competitive Anxiety. In Y. Hanin (Ed). Emotions in Sport. Champaign. Human Kinctics, pp. 93-113.

Simón, M.A. (1997). Manual de psicología de la salud: fundamentos, metodología y aplicaciones. Madrid: Biblioteca Nueva.

Sonnenschein, 1. (1995). Das Kolner Psychoregulationstraining. Ein Handbuch fur Trainingsleiter: Koln:bps.

Weinber, R. y Gould, D. (2000). Foundations of Sport and Exercise Psychology. Champaign: Human Kinetics. 


\section{Sugerencias para el afrontamiento del estrés}

Sin pretender ofrecer una propuesta exhaustiva y muchos menos definitiva que oriente a directores y directoras en el afrontamiento del estrés, señalamos algunas posibilidades que facilitarían los procesos de ajuste de las personas en situaciones de vivencia de estrés. En primer lugar, el tratamiento del cstrés requiere que se produzca un cambio en nucstra forma de pensar y de comportarnos ante las situacioncs y circunstancias de la vida que provocan tensión. Siguiendo a Mill (1987), las personas que enfrentan cxitosamente el estrés, se caractevizan por asumir la vida cotidiana con una actitud saludable, caracterizada por el optimismo, la motivación de logro, el sentimiento de control y autoeficacia. Es importante tener presente que, las situaciones en que se vive el estrés no deben ser vistas como una amenaza, sino más bicn, como un desafió que exige ponerse en acción, trabajar con el fin de resolver el problema (Lazarus, 1986).

De manera que, mantener una actitud positiva y optimista es fundamental en el afrontamiento del cstrés. Asimismo, es preciso hacer consciencia de los patrones de comportamiento inadecuados que poseemos y modificarlos en función de una mejor calidad de vida.

El afiontamicnto del cstrís demanda cl uso consciente de las capacidades cognoscitivas y de comportamiento, vinculadas con cl desarrollo de una actirud propositiva, de bísegueda de soluciones y de enfrentamiento decidido del problema. Algunas de las estrategias que se pucden scguir en este proceso son:

- Identificar y concentrarsc cn los aspectos relevantes de la situación que nos acerquen a una posiblc solución. Esto significa afrontar el problema de maneril activa, lo que implica csfucrzos cognoscitivos y conductuales para mancjar directamente la situación en que sc cstá viviendo estrés. Afrontar cl estrés de mancra constructiva. significa también, buscar información rclativa a la situación que se cstá viviendo y' definir alternativas positivas de intervención.

- Mantencr cl control sobre el acompañante orgánico y cmocional asociado con la vivencia del estrés. En este contexto, se pucde cchar mano de distintas técnicas de rclajación existentes, por cjemplo, la rclạjación muscular progrcsiva, el entrenamiento autogénico, la meditación, el cjercicio físico, entre otras. 
- Trabajar sobre los factorcs que agudizan la vivencia del estrés, por cjemplo, ciertos rasgos asociados con el patrón de compottamicnto tipo A, como las explosiones de ira. En este contexto y aunque solo to mencionemos, contamos con enfoques terapéuticos como el Racional Emolivo (Ellis y Eliot, 1980), la Terapia Cognitiva de Beck (1979), la estrategiade Inoculación del Estrés de Mcichenbaum (1977), entreotros.

- Generar una red de apoyo social con la que se pueda contar en momentos de vivencia del esırés, de manera que tengamos acceso directo y pronto a diversas opinioncs sobre cl problema cn cuestión y a descargar iensión. En este sentido, son numerosas las evidenciasen cuanto al papel protoctor que tiene cl apoyo social durante el afrontamiento del estrés (Moss, 1988). Contar con amigos, amigas, personas cercanas, ayuda a desamollar el sentimiento subjetivo de pertenencia, de ser aceptado, querido o neccsitado lanto poruno mismo como por lo que uno puede hacer. Entre las aclividades que podrian servir a estos propósitos lenemos: Organizar cxcursiones a la naturaleza, ir al teatro, departir tardes de café, lodas cstas actividades en pequeños grupos.

- Revisar y modificar, de ser necesario, las autovcrbalizaciones negativas, sustiluyéndolas por diálogos intcmos afirmativos y positivos.

- Hacer cjercicio físico representa una de las altcmalivas más prácticas y cficaces en ci afrontamiento del estrés. Sin embargo, es recomendable haccrlo periódicamente y en grupo, de manera que promovamos la salud intcgralmentc, mejorando nuestra capacidad funcional orgánica, psicológica y social. Es de fundamental importancia considcrar que, cl inicio de cualquicr programa de ejcrcicio físico debe scr precedido de una valoración médica competente. Las posibilidades Icrapecuticas del ejercicio físico se asocian con protocolos que implican realizar actividad física al menos 3 veces por semana, 30 minutos cada vez. como mínimo y con una intensidad moderada, esto significa cntre un 60 y $70 \%$ de la capacidad de consumo máximo de oxígeno.

En criterio de Mostofsky y Zaichkowsky (2002) y Sánche (1996) cl cjercicio físico tienc un valor terapéutico importante por cuanto:

- Aumenta la sensación psicológica de biencstar

- Mejora cl cstado anímico de la persona

- Fortalece la autoestima

- Favorcce la reducción de síntomas depresivos 
- Favorece la posibilidad de interacción

- Favorece el autocontrol emocional, fortalece la voluntad para el esfuerzo, la disciplina y la superación

- Promueve la adquisición de nuevas pautas de conducta (prosociabilidad, cooperación, amistad y socioempatia)

Debemos agregar que el empleo de cualquier estrategia para el afrontamiento del estrés requiere, en primer lugar, un esfuerzo sistemático y sostenido para aplicarlas en la vida diaria. En segundo lugar, cada persona ha de trabajar para hacer suyas las distintas estrategias a partir de sus vivencias $y$ reflexiones, y configurar una forma particular para su empleo según características personales y tomando en consideración el contexto dentro del cual esas estrategias se han de emplear.

\section{Referencias}

Beck, A. (1979). Cognisive therapy of behaviormodification. New York: Guilford Press.

Ellis, A. y Elliot, A. (1980). Terapia racional emotiva. México: Pax.

Meichenbaum, D. (1977). A cognitive behavior modificativin. New York: Plenun.

Mill, J. (1987). Cómo superar el estrés. Bilbao: Deusto.

Moss, R.H. (1988). Life stressors and coping resource influence health and wellbeing. Psychological assessment, 4, pp. $133-158$.

Mostoisky, D.L. \& Zaichkowsky L.D. (2002). Medical and psychological aspects of sport and exercise. Morgentown. FIT.

Sánche\%. F. (1996). La actividad fisica orientada hacia la salud. Barcelona: Nueva Deciada. 